\title{
Wissenschaftlicher Förderungspreis 2019: Ekaterina Then, Venenklinik Bellevue Kreuzlingen
}

\section{Wertigkeit des Clippings der Vena ovarica bei Patientinnen mit Varikose der unteren Extremitäten}

Das pelvine Stauungssyndrom (Pelvic Congestion Syndrom $=$ PCS) erfuhr in den vergangenen 20 Jahren zunehmende Aufmerksamkeit. Man geht davon aus, dass rund $15 \%$ der Frauen im reproduktionsfähigen Alter (18-50 Jahre alt) an einem PCS leiden. Das PCS ist definiert als chronisch persistierender Beckenschmerz bei Frauen während einer Dauer von mindestens 6 Monaten. Ursächlich wird eine „pelvine venöse Insuffizienz“ (PVI) postuliert. Die PVI wird am häufigsten durch einen Reflux in der Vena ovarica sinistra nach einer oder mehreren Schwangerschaften verursacht.

Durch die Erschöpfung der pelvinen Drainagekapazität können sich an den Beinen Varizen mit spezifischen morphologischen Mustern ausprägen.

Dieser Symptomen-Komplex, für welchen das Ärzteteam der Venenklinik Bellevue Kreuzlingen den Ausdruck Ovarialveneninsuffizienz-Syndrom (OVIS) prägte, betrifft nach deren Statistik 7,4\% aller Frauen unabhängig vom Alter.

Bei einem insgesamt sehr variablen Beschwerdebild sind früh auftretende postoperative Rezidivvarizen perinealen, subglutaealen oder pudendalen Ursprungs charakteristisch. Beschwerden im Kontext dieser Varizen stellen oft den primären Behandlungsgrund dar und sind oft der einzige klinische Hinweis auf das Vorliegen einer venösen Überlastung im Beckenbereich. Nach Sicherung der Diagnose eines OVIS mittels Schnittbildverfahren (MR-Phlebographie) kann der Ovarialvenenreflux durch eine Sanierung des proximalen Insuffizienzpunktes entweder chirurgisch mittels laparaskopischen Clippings oder radiologisch mit gezielter Coil-Embolisation behandelt werden. Zu beiden Therapiemethoden gibt es bis heute keine publizierten grösseren Fallserien mit Langzeitverlauf.

Im Zeitraum von 2009 bis 2017 wurden an der Venenklinik Bellevue Kreuzlingen total 107 Patientinnen mit OVIS chirurgisch mittels laparoskopischen Clippings der linken Vena ovarica behandelt. In der vorliegenden Studie sollen alle Patientinnen erneut aufgeboten und nachuntersucht werden.

Primäres Studienziel: Häufigkeit von Rezidivvarizen der unteren Extremitäten nach Clipping der V. ovarica sinistra (Klinik und Duplexsonographie). Verlaufs-MR-Phlebographie: Anatomischer Zusammenhang allfälliger Rezidivvarizen mit dem Beckenvenensystem im Langzeitverlauf nach Clipping.
Sekundäres Studienziel: Dokumentation des Einflusses des Clippings der V. ovarica sinistra auf die Bein- und Beckenbeschwerden im Rahmen des Pelvic Congestion Syndroms, mittels Fragebogen und Score. Verlaufs-MR-Phlebographie: Anatomischer Zusammenhang allfälliger Symptome mit dem Beckenvenensystem im Langzeitverlauf nach Clipping.

Der Vorstand der Schweizerischen Gesellschaft für Phlebologie hat dieses Projekt unter mehreren hochkarätigen wissenschaftlichen Projekt-Bewerbungen ausgewählt. Wir gratulieren der Preisträgerin sehr herzlich und warten gespannt auf die Resultate, welche die Erstautorin zum Abschluss als Vortrag an einem der nächsten offiziellen Tagungen der SGP präsentieren und selbstverständlich auch publizieren wird.

\section{IMPRESSUM}

Verantwortlich für Mitteilungen der SGP:

Prof. Dr. med. Jürg Hafner, Zürich 